$16^{\text {th }}$ International Congress of Metrology, 18001 (2013)

DOI: $10.1051 /$ metrology/201318001

(C) Owned by the authors, published by EDP Sciences, 2013

\title{
EMRP-ENV03: Traceability for surface spectral solar ultraviolet radiation
}

\author{
Jimmy Dubard ${ }^{1, \mathrm{a}}$, Thierry Valin ${ }^{1}$, Romain Etienne ${ }^{1}$ and Géraldine Ebrard ${ }^{2}$ \\ ${ }^{1}$ LCM (LNE-CNAM), 29 avenue Roger Hennequin, ZA Trappes-Elancourt, 78197 Trappes, France \\ ${ }^{2}$ LNE, 29 avenue Roger Hennequin, ZA Trappes-Elancourt, 78197 Trappes, France
}

\begin{abstract}
The ENV03 Solar UV project is aimed at improving the traceability of solar UV spectral irradiance measurement. This is achieved by developing new characterization methods and devices (stray light improved array spectroradiometer, Fourier Transform Spectrometer). The workpackages of the project are presented. We describe the output of two tasks in which the LNE is involved: the realization of a software for evaluating the measurement uncertainty using Monte Carlo technique, and the development of a reduced stray light array spectroradiometer fitted with band-pass filters to measure the UV spectral irradiance with an uncertainty $<2 \%$.
\end{abstract}

\section{Introduction}

The quantification of UV radiation at the earth surface requires accurate measurements of global solar spectral UV irradiance in order to understand long-term trends in this parameter, of the order of $2 \%$ per decade over Europe, due to atmospheric changes. Measurements with uncertainties of around $1 \%$ to $2 \%$ are needed to detect such decadal changes.

The current knowledge on spectral solar UV radiation is limited to very few places worldwide where spectral solar UV monitoring instruments (scanning spectroradiometers) are located. Large-scale deployment of such instruments is limited by the required manpower and infrastructure to guarantee an adequate level of uncertainty. Moreover the rapid temporal variation of UV radiation due to atmospheric conditions (e.g. clouds) requires fast spectroradiometers with stray light rejection of at least 10-6 to reliably sample the whole solar UV spectrum between $300 \mathrm{~nm}$ and $400 \mathrm{~nm}$.

Fast spectroradiometers such as today array spectroradiometers are not suitable for solar UV measurements without complex correction methodologies due to the large dynamic range of the solar UV radiation between 300 and $400 \mathrm{~nm}$ and the significant stray light contamination of these instruments.

There is a need to significantly enhance the reliability of spectral solar UV radiation in the wavelength range 300 $\mathrm{nm}$ to $400 \mathrm{~nm}$ measured at the earth surface using such array spectroradiometers and the European Metrology Research Program EMRP-ENV03 "Traceability for surface spectral solar ultraviolet radiation" is aimed to fulfill this need with an uncertainty of less than $2 \%$.

\footnotetext{
${ }^{\mathrm{a}}$ Corresponding author: jimmy.dubard@lne.fr
}

We present the objectives of the project and the work to be performed. We focus on LNE contributions particularly to workpackages (WP):

- WP2 task 2.2 about the evaluation of the uncertainty of the measurements performed with array spectroradiometers

- WP4 task 4.4 about the development and characterization of an array spectroradiometer fitted with tailored bandpass filters for improved stray light rejection.

\section{ENV03 SolarUV project}

The ENV03 Solar UV project aims to improve the uncertainty of the solar UV spectral irradiance. Four technical work packages are dedicated to developing new characterization methods and devices (stray lightimproved array spectroradiometers, Fourier Transform Spectrometers) to provide traceable solar UV irradiance measurements with an uncertainty $<2 \%$.

The project is coordinated by SFI-Davos and involves 7 NMIs (Aalto, CMI, EJPD, INRIM, LNE, PTB, VSL), 3 unfunded partners and 2 REG.

\subsection{WP1: Spectral irradiance traceability}

The aim of this work package is to shorten the traceability chain of solar UV measurements in the wavelength range $300 \mathrm{~nm}$ to $400 \mathrm{~nm}$ to the SI unit and to reduce the associated transfer uncertainties. The goal is to develop uncertainties in the field comparable to those currently achieved only for primary spectral irradiance scale realisations at NMI level, i.e., at the level of $\pm 1 \%$ to $\pm 2 \%$ at $\mathrm{k}=2$. This workpackage will: 
- Develop a detector based spectral irradiance traceability chain. The improved European Reference spectroradiometer QASUME will be calibrated directly against a reference detector traceable to an absolute radiation standard using a tuneable laser facility. No intermediate source-based transfer standards will be required in this traceability chain.

- Develop two UV LED-based transfer standards: These are compact, stable and portable monitoring devices based on UV-LEDs, with two entrance optics geometries that will be used to maintain the spectral calibration of spectroradiometers while deployed at the UV monitoring sites.

- Develop a high output metrology (laser) light source for field calibrations with a spectral power distribution free of impurity features and spectrally flat to a level of a few percent over the solar UV range. This laser-based device has high radiation output and can be used in the far-field region (in contrast to the UV-LED devices) and thus calibrate spectroradiometers with diverse input optics.

\subsection{WP2: Array spectroradiometer characterisation}

The aim of this workpackage is to develop new characterisation techniques for UV array spectroradiometers addressing the most relevant sources of errors. Measuring the UV part of the solar spectrum requires a high dynamic range due to the sharp cut off of the solar spectrum in the UVB-range by ozone absorption. The current limitations of array spectroradiometers make them unsuitable for solar UV measurement. The main effects to consider when characterising UV array spectroradiometers are spectral stray light, bandwidth, wavelength accuracy and linearity. Quantifying these effects is not only fundamental for calculating the measurement uncertainty but it also allows correcting for the instrument imperfections and thus decreases the overall uncertainty of measurements.

This workpackage will:

- Identify requirements of array spectroradiometers for solar UV measurements in the range $300 \mathrm{~nm}$ to $400 \mathrm{~nm}$, and the best measurement procedures. This will be reorted in "A guide to measuring solar UV spectra using array spectroradiometers".

- Develop a complete model for uncertainty calculation of array spectroradiometers that will include all relevant parameters including effects of correlations among spectral values.

- Characterise and correct UV array spectroradiometers for in-range and out-of-range stray light effects.
- Develop an algorithm to transform measured solar spectra onto a uniformly spaced wavelength grid with a nominal spectral resolution. It will allow correcting band pass effect of even low resolution instruments by using a high-resolution solar reference spectrum.

- Develop two wavelength characterisation devices for array spectroradiometers: a Fabry-Perot multilayer structure working in the UV range that will enable the characterisation of the wavelength scale at the \pm 0.01 $\mathrm{nm}$ uncertainty level, and a polarisation gradient filter concept usable for field applications.

- Compare three linearity characterisation methods based on complementary techniques.

\subsection{WP3: Improvement of reference spectroradiometers}

Currently even the best UV solar reference spectroradiometers do not provide sufficient accuracy for long-term analysis of atmospheric changes. Total uncertainty of these systems for in field measurement of solar spectral irradiance in UV reaches the level of $5 \%$.

For monochromator-based scanning spectroradiometers a significant part of this uncertainty is due to the photomultiplier tubes (PMT) used as detector: nonlinearity, memory effect and poor long-term quantum efficiency stability.

The entrance optics for global UV measurements also need to be improved to reduce diurnal variations arising from the changing solar elevation and the ratio between diffuse and direct solar irradiance; the latter task will be accomplished in WP4.

The following activities will be undertaken:

- Develop a new solid-state detection system (SSDS) suitable for replacing a PMT detector. This solution is supported by recent improvements of photodiodes manufacturing process and readout electronics that have made possible to consider semiconductor detectors and switched integrator amplifiers a valid alternative to PMT.

- Modify and validate the portable reference scanning spectroradiometer QASUME. The selected SSDS will be fitted in place of the PMT along with an improved global entrance optics developed in WP4 . Validation will occur in the lab and the field. The improved QASUME will be used for disseminating the improved UV irradiance scale to stakeholder instruments through the intercomparison in Davos (WP5).

- Adapt and validate a commercially available Fourier Transform Spectroradiometer (FTS) for solar UV irradiance measurements. The aim is to offer high wavelength accuracy and perform fast measurements under rapidly varying environment conditions ("cloudy sky"). 


\subsection{WP4: New technologies}

The aim of this workpackage is to develop new techniques, technologies and equipment that can be used to improve the uncertainties of solar UV measurements. Currently the commercially available cost-effective spectroradiometers have a number of limitations. They do not achieve the required sensitivities; deviations of at least $20 \%$ in solar UV irradiance measurements at 310 $\mathrm{nm}$ are currently observed due to: a non-ideal cosine response of the global entrance optics and inadequate stray light rejection in the UV wavelength range particularly in the UV-B range

This WP aims to resolve these issues by:

- Developing a hyperspectral sensor for the determination of spectral UV sky radiance. This will be used as ancillary instruments to correct spectral measurements from current spectroradiometers with traditional (nonoptimised) global entrance optics and by spatial integration to provide also the diffuse spectral irradiance as a traditional imaging spectroradiometer.

- Developing new global entrance optics (diffusers) with improved angular responses.

- Developing array spectroradiometers with optimised stray light rejection of $10^{6}$ (for measuring solar UV irradiance). Two different approaches to stray light rejection will be investigated; Task 4.3 investigates the use of adaptive optics, whilst task 4.4 investigates filtering, both will be thoroughly characterised by the tools developed in WP1 and WP2.

\section{LNE contribution to ENV03 project}

LNE is involved into two WP within the ENV03 project. It is charge of task 2.2 about the uncertainty evaluation of measurement performed with array spectroradiometers and task 4.4 about the improvement of the stray light rejection of a modified array spectroradiometer using band-pass filters.

\subsection{Array spectroradiometer measurement uncertainty evaluation}

Accurate measurement of the UV spectral irradiance with an array spectroradiometer required some data corrections: linearity, wavelength scale, stray light... These complex corrections need to be taken into account for the evaluation of the uncertainty associated to the measurements. We propose to use the Monte Carlo (MC) technique described in the part 3 of the GUM [1] for the uncertainty evaluation [2]. The MC technique requires a well defined measurement model.

For the measurement of the solar UV spectral iarradiance two steps are necessary:

- The calibration of the spectroradiometer

- The measurement of the natural sun light
For the spectroradiometer calibration using a standard lamp the measurement model is the following.

$$
S_{S t d, i}=\frac{\left(M_{S t d, i}-M_{D S t d, i}\right) C_{L i n}}{T_{I n t, S t d}} C_{C o s} C_{\lambda} \quad(\text { counts } / s)
$$

where:

$M_{\text {Std,i }}$, the signal for pixel " $i$ " corresponding to $\lambda_{i}$ when measuring the standard lamp

$\mathrm{M}_{\mathrm{DStd}, \mathrm{i}}$, the measured dark signal for pixel " $\mathrm{i}$ "

corresponding to $\lambda_{\mathrm{i}}$ when measuring the standard lamp $\mathrm{T}_{\text {Int,Std }}$, the integrating time when measuring the standard $\mathrm{C}_{\mathrm{Lin}}$ is the correction due to non-linearity response of CCD elements

$\mathrm{C}_{\mathrm{Cos}}$ is the correction due to non-perfect cosine response $\mathrm{C}_{\lambda}$ is the correction due to the wavelength scale calibration

Because array spectroradiometer is based on a single monochromator stray light correction should be applied. The true signal $\mathrm{S}_{\text {True, } i}$ is obtained from the measured signal $\mathrm{S}_{\mathrm{Std}, \mathrm{I}} \mathrm{using}$ the equation 2 :

$$
S_{S t d, i}=S_{\text {True }, i}+\sum_{j} S_{\text {True }, j} d_{i, j}
$$

Where $d_{i, j}$ is the stray light contribution arising from light at $\lambda_{j}$. Equation 2 can be written in the matrix form:

$$
S_{\text {True }}=(1+D)^{-1} S_{S t d}
$$

$\mathrm{D}$ is the stray light correction matrix and " 1 " is the matrix unity. D contains only non-zero off diagonal elements that are small compare to " 1 ". Therefore $(1+D)^{-1}$ reduces to $(1-\mathrm{D})$.

Finally for each wavelength " $i$ " the calibration factor of the array spectroradiometer is given by:

$$
C A L_{i}=\frac{S_{\text {True }, i}}{E_{S t d, i}}
$$

Where $E_{S t d, i}$ is the spectral irradiance of the standard lamp.

For the measurement of the sun light the same measurement model described in equations 1 to 3 is applied. The spectral irradiance of the sun light $E_{\text {Sun, } \mathrm{i}}$ is given by:

Where $\mathrm{S}_{\mathrm{Sun}, \mathrm{i}}$ is the $E_{\text {Sun }, i}=\frac{S_{\text {Sun }, i}}{C A L_{i}}$ stray light corrected measured signal.

The uncertainty evaluation is performed in steps that correspond to the equations 1 to 5 . The probability distribution function PDF of all uncertainty components are defined. Correlation between input quantities are taken onto account. For instance uncertainty due to the stray light correction is evaluated based on equation 6 : 


$$
S_{\text {True }, i}=\sum_{j} S_{S t d, j}(1-D)_{i, j}
$$

$(\mathrm{j}=1$ to $\mathrm{N}$ ), where $\mathrm{N}$ is the number of wavelengths taken into account.

The $\mathrm{S}_{\mathrm{Std}, \mathrm{j}}$ elements are correlated as well the (1-D) $)_{\mathrm{i}, \mathrm{j}}$ elements. The multivariate Gaussian algorithm [1] is used to evaluate the uncertainty associated to these correlated input quantities.

A software using Matlab is under development to evaluate the uncertainty. The validation will be performed using data from the various tasks of WP2. A guideline will be issued to allow a proper use of the software.

\subsection{Development of an improved stray light rejection array spectroradiometer}

Array spectroradiometer suffers from stray light contribution particularly when weak signal needs to be measured while strong signal is present. Today, technique has been developed to correct the signal from stray light [3] based on a tedious characterization of the spectroradiometer [4]. However uncertainty on this characterization can lead to large uncertainty on the sun spectral irradiance measurement particularly on the 280 $\mathrm{nm}-300 \mathrm{~nm}$ spectral range.

To reduce the contribution of stray light LNE proposes to improve this characteristic of an array spectroradiometer by adding proper band-pass filters. The array spectroradiometer is a VS140 type from the manufacturer Jobin Yvon. The modified spectroradiometer is shown on figure 1 .

Figure 1. Schematic of the modified spectroradiometer

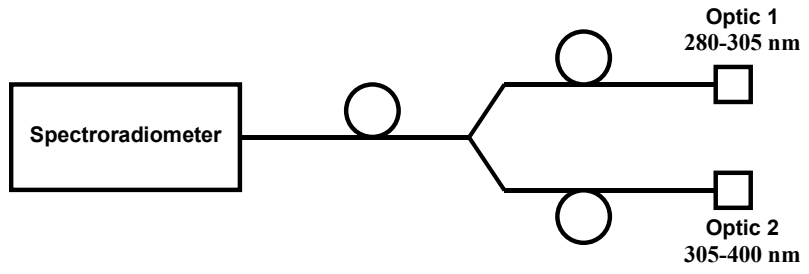

To measure the spectral irradiance over the $280 \mathrm{~nm}-400$ $\mathrm{nm}$ spectral range two optics are necessary. One for the $280 \mathrm{~nm}-305 \mathrm{~nm}$ range and one for the $305 \mathrm{~nm}-400 \mathrm{~nm}$ range.

Figure 2 shows the transmittance measurement of the combinations of band-pass filters that can be used for the $280 \mathrm{~nm}-305 \mathrm{~nm}$ range. From these measurements the association of the SEMROCK $292 \mathrm{~nm}$ interference filter and the $3 \mathrm{~mm}$ thick UG11 filter is the best solution as it will suppress the light $>310 \mathrm{~nm}$.
Figure 2. Transmittance of filters for the Optic1 channel

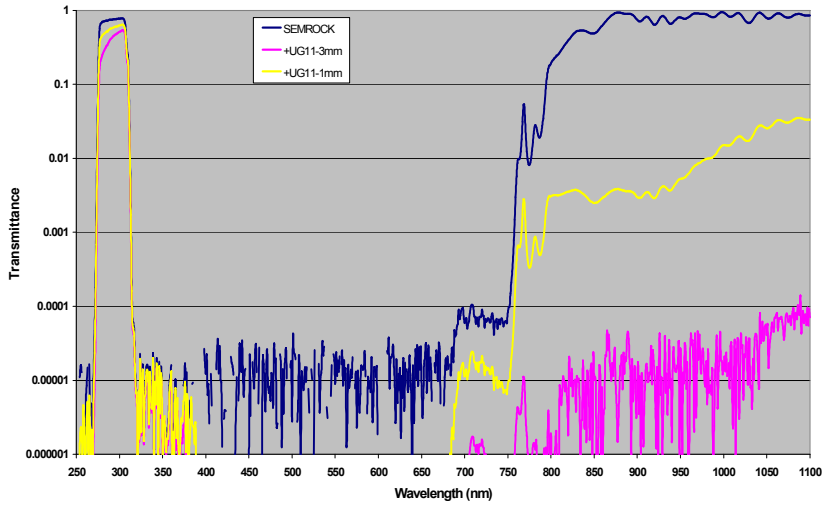

Figure 3 shows the transmittance measurement of the filters for the $305 \mathrm{~nm}-400 \mathrm{~nm}$ range. The UG11RB filter is interesting because of the strong attenuation in the visible and near infrared regions. However the cut-off wavelength in the upper part of the UV region is $<400$ $\mathrm{nm}$. The best choices are the $0.5 \mathrm{~mm}$ thick UG11 filter or the $1 \mathrm{~mm}$ thick UG1 filter.

Preliminary characterization of the spectroradiometer have been performed. This includes: linearity, stray light. The results of the time integration linearity is shown on figure 4.

A complete characterization of the stray light will be performed at PTB beginning of 2014 .

Figure 3. Transmittance of filters for the Optic2 channel

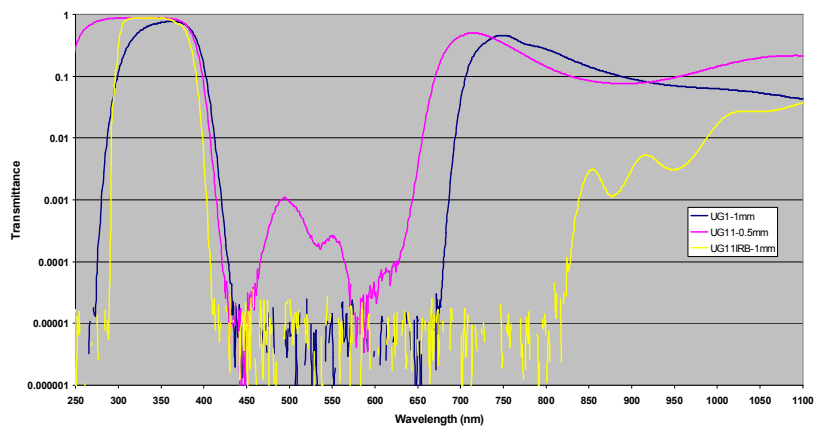

Figure 4. Time integration linearity characterization

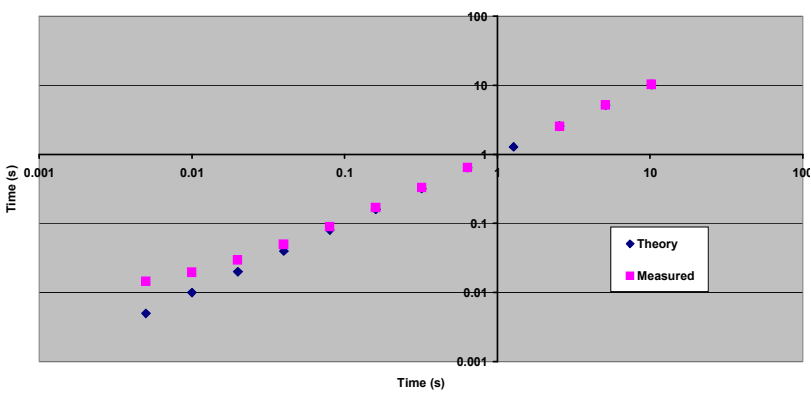


The modified spectroradiometer will take part to the comparison that planned in July 2014 in Davos, Switzerland.

\section{Conclusion}

The aim of the ENV03 is to improve the uncertainty of the measurement of the solar UV spectral irradiance. LNE contributes on two subjects: the evaluation of the uncertainty of the measurement using array spectroradiometers and the development of a reduced stray light array spectroradiometer.

The uncertainty will be evaluated using the MonteCarlo technique. A software will be edited along with a guideline.

Band-pass filters will be added to a UV optimized array spectroradiometer to improve the stray light rejection. The filters are characterized and stray light will be evaluated. The spectroradiometer will participate to a comparison in July 2014 and we expect that the improvement of the stray light will allow us to reach an uncertainty $<2 \%$.

\section{Acknowledgements}

The EMRP is jointly funded by the EMRP participating countries within EURAMET and the European Union.

\section{References}

1. ISO/IEC Guide 98-3/Suppl. 1 "Propagation of distributions using a Monte Carlo method", (2008)

2. A-F Obaton, J. Lebenberg, N. Fischer, S. Guimier, J. Dubard, „Two procedures for the estimation of the uncertainty of spectral irradiance measurement for $U V$ source calibration", Metrologia, 44, (2007)

3. Y. Zong, S-W. Brown, B. C. Johnson, K.R. Lykke, Y. Ohno, "Simple spectral stray light correction method for array spectroradiometer", Applied optics 45, 6 (2006)

4. S. Nevas, G. Wübbeler, A. Sperling, C. Elster, A. Teuber, ,Simultaneous correction of bandpass and straylight effect in array spectroradiometer data", Metrologia, 49, (2012) 